\title{
Aggregated Preference Value Analysis of Small Satellite Launch Opportunities*
}

\author{
Mengying ZHANG, ${ }^{1)}$ Qin XU, ${ }^{2)}$ and Qingbin ZHANG ${ }^{1) \dagger}$ \\ ${ }^{1)}$ College of Aeronautics and Astronautics, National University of Defense Technology, Changsha, Hunan 410073, China \\ ${ }^{2)}$ School of Mechanical, Aerospace and Civil Engineering, The University of Manchester, Manchester M13 9PL, UK
}

\begin{abstract}
Due to the lack of matched dedicated small satellite launch vehicles, launch opportunities as a rideshare or piggyback using a highly reliable launch system has become increasingly important for small satellite developers. The objective of this paper is to provide an aggregated value method as a strategy for developers to evaluate launch opportunities, as well as an approach for the launchers to capture the market dynamics. Based on an up-to-date launch record, a reliable launch database for multi-attribute evaluation is established. Efforts are made to quantify the abstract and concrete attributes of launch systems. An aggregated preference value model is developed, translating different inherited capabilities of launch systems into integrated preference value as a reference for decision-making. The preference values of the launch opportunities of different launch vehicles are explored in a case study by the method proposed, and its feasibility and applicability for small satellite launch system evaluation tasks is validated.
\end{abstract}

Key Words: Small Satellite, Preference Function, Launch Opportunity, Decision Making

\section{Introduction}

In recent years, small satellites have been playing an increasingly more significant role in space activities as the result of growing government interests, industry initiatives and technological advancements (Fig. 1). ${ }^{1,2)}$ However, the miniaturization of satellites has not been fully followed in parallel with the reduction in payload capacity of our launch vehicles, or in other words, the development of dedicated small satellite launch vehicles. ${ }^{3)}$ On one hand, the motivation of lower cost and risks has stimulated an upward demand and interest in developing dedicated launch vehicles: at least 20 were in development by the year 2015. ${ }^{3-5)}$ On the other hand, the current market still shows an intention to use conventional launch vehicles, where more satellites are being integrated on larger vehicles. One recent case is that India has set a record by successfully launching 104 satellites in a single trial on 15 February 2017.6) Under these circumstances, before dedicated vehicles reach their technical maturity, small satellite developers need to seek rideshare or piggyback opportunities as secondary payloads. Thus, no matter how quickly the market trend changes, the success of small satellite projects largely depends on the performance of launch systems and the acquisition of launch opportunities.

As the launch market continues to heat up, some launch vehicles become less desirable in the competition while others become extremely popular. Although there are still doubts on whether this market trend represents the actual de-

(C) 2018 The Japan Society for Aeronautical and Space Sciences

*Presented at the 31st International Symposium on Space Technology and Science, 3-9 June 2017, Matsuyama, Japan.

Received 22 June 2017; final revision received 31 October 2017; accepted for publication 12 December 2017.

†Corresponding author, qingbinzhang@sina.com mand from both technical and economical perspectives, analysis of known launch histories is necessary to uncover current key concerns from different aspects.

From the demand side, optimized decisions are required for practical considerations, including customer affordability, system reliability, technology maturity and the availability of launch opportunities. From the provider side, such intentions are crucial references for launch vehicles to adapt to the dynamic trend in the emergence of market imbalance.

In order to provide a more comprehensive strategy for satellite developers to make decisions and benefit the providers by enabling them to understand market trends, an aggregated preference value method for the overall capability evaluation of small satellite launch opportunities is proposed in this paper. Based on historical launch records, efforts have been made to update a launch vehicle database established in our previous research, which serves as the performance database for multi-attribute decision-making. A Bayesian analysis of the future success rate of current active launch vehicles is performed to estimate their predicted reliability. The concept of availability of launch opportunities is established and quantified. Focusing on multiple concerns towards the capabilities of launch vehicles, an aggregated preference value model is developed, allowing for a comprehensive decision-making process that integrates developers' experiences and technical indicators. The preference values of the launch opportunities of currently active launch vehicles are explored in a case study using the method proposed. The results validate that this analysis is capable of small satellite launch system evaluation and supports the decisionmaking process. 


\section{Launch Capability Analysis}

\subsection{Updated launch vehicle database}

The launch vehicle database established in our previous research is updated with the launch data from the International Reference Guide to Space Launch Systems, ${ }^{8)}$ Space Mission Engineering: The New SMAD, ${ }^{9}$ and online space launch system data. ${ }^{10-12)}$ Note that although the cost per kilogram actually delivered to orbit may be different from that advertised due to the amount of wasted payload capacity every launch, ${ }^{13)}$ we still use the total cost divided by the maximum payload as the metric for unit cost. Based on the previous version, ${ }^{14)}$ one new attribute, namely average launch cycle, is added to the database to reveal the general frequency of the launch activities. Note that all of the launch systems presented here are classified into two categories following the classification in The New SMAD according to their payload capability to LEO; namely, small launch systems $(<5,000 \mathrm{lb})$ and medium and intermediate launch systems $(<12,000 \mathrm{lb}$ and $<25,000 \mathrm{lb}$, respectively). By 31

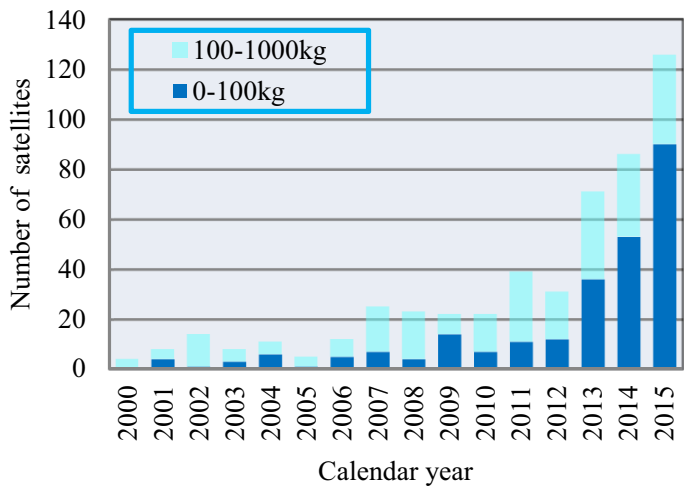

Fig. 1. Small satellite launch activity history recorded in the UCS satellite database. $^{7)}$
January 2017, different fundamental concerns of the selected launch systems are exhibited in Table 1 and Table 2, to cover the most significant performance indices of launch systems.

Please notice that all of the heavy launchers (e.g., LEO payload capability $>25,000 \mathrm{lb}$ ) are excluded in the proceeding analysis. This kind of launch systems is mainly designed for heavy transportation missions such as cargo delivery to the International Space Station (ISS) or deep-space exploration tasks. Generally, heavy launch vehicles are less appealing for launching small satellites as compared to dedicated vehicles, although they are capable. Currently, heavy launch vehicles are still not one of the mainstream means for launching small satellites into LEO due to the demand (i.e., small market size). Therefore, despite some well-known heavy launch systems that have executed small satellite missions, only small, medium and intermediate launch systems are fully discussed in this study.

\subsection{Availability analysis}

\subsubsection{Availability expectation analysis}

In the reliability theory, availability is typically measured as a factor of reliability. When considering the case of launch opportunities, availability is apt to be a factor independent of reliability. The formal definition and main extended meaning of availability - the quality of being able to be used or obtained and the state of being otherwise unoccupied ${ }^{15)}$-indicate that this word defines a free and accessible approach to some subject. There are many ways of quantifying this attribute. For example, as is described in the definition of "achieved availability $A$," it is calculated by the mean time between maintenance (MTBM) action, and the mean maintenance time (MMT). ${ }^{16)}$

$$
A=\frac{\mathrm{MTBM}}{\mathrm{MTBM}+\mathrm{MMT}}
$$

Table 1. Small launch systems (small launch vehicles are identified by their payload capabilities in pounds to LEO $(<5,000)$ ).

\begin{tabular}{|c|c|c|c|c|c|c|c|c|}
\hline \multirow{2}{*}{ Vehicle } & \multirow{2}{*}{ Country } & \multicolumn{2}{|c|}{ Unit cost $(\mathrm{FY} 2010 \$ \mathrm{~K} / \mathrm{kg})$} & \multirow{2}{*}{$\begin{array}{c}\text { Total } \\
\text { launches }\end{array}$} & \multirow{2}{*}{$\begin{array}{l}\text { Success } \\
\text { rate }(\%)\end{array}$} & \multirow{2}{*}{$\begin{array}{c}\text { Average } \\
\text { launch cycle (d) }\end{array}$} & \multirow{2}{*}{$\begin{array}{l}\text { Last launch date } \\
\text { (DDMMYYYY) }\end{array}$} & \multirow{2}{*}{ Status } \\
\hline & & LEO & $\mathrm{SSO}$ & & & & & \\
\hline Athena 2 & USA & 15.8 & 28.1 & 3 & 66.67 & 313 & 24/09/1999 & Retired \\
\hline Delta 2000s & USA & 31.1 & 41.5 & 44 & 97.73 & 66 & $06 / 10 / 1981$ & Retired \\
\hline Epsilon & Japan & 31.7 & 84.4 & 2 & 100.00 & 1193 & $20 / 12 / 2016$ & Active \\
\hline Falcon 1 & USA & 10.4 & 17.0 & 5 & 40.00 & 302 & $14 / 07 / 2009$ & Retired \\
\hline Kosmos 3M & Russia & 12.1 & 23.7 & 446 & 95.07 & 35 & $27 / 04 / 2010$ & Active \\
\hline $\mathrm{KZ}$ & China & 8.7 & / & 3 & 100.00 & 601 & $09 / 01 / 2017$ & Active \\
\hline Minotaur I & USA & 35.9 & 68.7 & 11 & 100.00 & 505 & $20 / 11 / 2013$ & Active \\
\hline Minotaur IV & USA & 13.3 & 22.0 & 5 & 100.00 & 131 & $27 / 09 / 2011$ & Active \\
\hline M-V & Japan & 37.7 & 74.6 & 7 & 85.71 & 403 & $22 / 09 / 2006$ & Retired \\
\hline Pegasus & USA & 43.1 & 100.4 & 43 & 87.88 & 256 & $15 / 12 / 2016$ & Active \\
\hline Rockot & Russia & 9.5 & 18.5 & 30 & 90.00 & 322 & $04 / 06 / 2016$ & Active \\
\hline Shavit 1 & Israel & 58.5 & / & 6 & 66.67 & 1127 & $06 / 09 / 2004$ & Active \\
\hline Shtil' & Russia & 15.7 & / & 2 & 100.00 & 2880 & $26 / 05 / 2006$ & Active \\
\hline START & Russia & 19.5 & 73.6 & 6 & 100.00 & 956 & $25 / 04 / 2006$ & Active \\
\hline Strela & Russia & 9.2 & 20.5 & 3 & 100.00 & 2016 & $19 / 12 / 2014$ & Active \\
\hline Taurus & USA & 18.8 & 35.9 & 9 & 66.67 & 771 & $04 / 03 / 2011$ & Active \\
\hline Taurus XL & USA & 28.5 & 52.6 & 3 & 33.33 & 1270 & $04 / 05 / 2011$ & Active \\
\hline Titan 2 & USA & 25.2 & 43.5 & 13 & 92.31 & 460 & $18 / 10 / 2003$ & Retired \\
\hline Vega & Italy & 17.8 & 25.1 & 8 & 100.00 & 251 & $05 / 12 / 2016$ & Active \\
\hline Volna & Russia & 11.2 & 39.3 & 5 & 40.00 & 944 & $06 / 10 / 2005$ & Active \\
\hline
\end{tabular}


Trans. Japan Soc. Aero. Space Sci., Vol. 61, No. 2, 2018

Table 2. Medium and intermediate launch systems (medium and intermediate launch vehicles are identified by their payload capabilities in pounds to LEO $(<12,000$ and $<25,000$, respectively) $)$.

\begin{tabular}{|c|c|c|c|c|c|c|c|c|}
\hline \multirow{2}{*}{ Vehicle } & \multirow{2}{*}{ Country } & \multicolumn{2}{|c|}{ Unit cost $(\mathrm{FY} 2010 \$ \mathrm{~K} / \mathrm{kg})$} & \multirow{2}{*}{$\begin{array}{c}\text { Total } \\
\text { launches }\end{array}$} & \multirow{2}{*}{$\begin{array}{l}\text { Success } \\
\text { rate }(\%)\end{array}$} & \multirow{2}{*}{$\begin{array}{c}\text { Average } \\
\text { launch cycle (d) }\end{array}$} & \multirow{2}{*}{$\begin{array}{l}\text { Last launch date } \\
\text { (DDMMYYYY) }\end{array}$} & \multirow{2}{*}{ Status } \\
\hline & & LEO & $\mathrm{SSO}$ & & & & & \\
\hline Ariane $44 \mathrm{~L}$ & Europe & 15.0 & 23.6 & 40 & 97.50 & 128 & $15 / 02 / 2003$ & Retired \\
\hline Atlas 2AS & USA & 15.4 & 25.6 & 30 & 100.00 & 135 & $31 / 08 / 2004$ & Retired \\
\hline Atlas 3 & USA & 13.2 & / & 6 & 100.00 & 343 & $03 / 02 / 2005$ & Retired \\
\hline Cyclone 2 & Ukraine & 8.4 & / & 106 & 99.06 & 128 & $24 / 06 / 2006$ & Retired \\
\hline Cyclone 3 & Ukraine & 6.9 & / & 122 & 93.44 & 95 & $30 / 01 / 2009$ & Retired \\
\hline Delta 2 & USA & 14.6 & 24.0 & 114 & 98.25 & 73 & $28 / 10 / 2011$ & Active \\
\hline Delta $2 \mathrm{M}$ & USA & 19.3 & 30.8 & 23 & 100.00 & 194 & $31 / 01 / 2015$ & Active \\
\hline Delta 4M & USA & 9.7 & 13.4 & 3 & 100.00 & 667 & $04 / 11 / 2006$ & Active \\
\hline Dnepr & Russia & 4.6 & 8.9 & 22 & 95.45 & 277 & $25 / 03 / 2015$ & Active \\
\hline Falcon 9 V1.0 & USA & 5.4 & / & 5 & 80.00 & 250 & $01 / 03 / 2013$ & Retired \\
\hline GSLV & India & 8.8 & / & 10 & 50.00 & 625 & 08/09/2016 & Active \\
\hline Long March 2C & China & 9.5 & 15.3 & 41 & 97.56 & 294 & $14 / 11 / 2014$ & Active \\
\hline Long March 2D & China & 5.9 & 15.8 & 31 & 100.00 & 297 & $21 / 12 / 2016$ & Active \\
\hline Long March 3A & China & 9.8 & 11.8 & 25 & 100.00 & 337 & $29 / 03 / 2016$ & Active \\
\hline Long March 3C & China & 11.3 & 15.8 & 15 & 100.00 & 224 & $22 / 11 / 2016$ & Active \\
\hline PSLV-G & India & 5.4 & 19.2 & 12 & 83.33 & 764 & $26 / 09 / 2016$ & Active \\
\hline PSLV-CA & India & 7.2 & 18.3 & 11 & 100.00 & 316 & $16 / 12 / 2015$ & Active \\
\hline PSLV-XL & India & 5.3 & 11.5 & 13 & 100.00 & 212 & $07 / 12 / 2016$ & Active \\
\hline Soyuz & Russia & 7.4 & / & 32 & 93.75 & 116 & $14 / 10 / 1976$ & Retired \\
\hline Soyuz 2 & Russia & 6.9 & 11.3 & 63 & 92.06 & 72 & $28 / 01 / 2017$ & Active \\
\hline Soyuz U & Russia & 7.8 & 12.7 & 785 & 97.32 & 20 & $01 / 12 / 2016$ & Active \\
\hline
\end{tabular}

If this definition is followed in the case of measuring the availability of launch opportunities, it would show a correspondence that MTBM is equal to the time spent on the launch activity, including its preparation time, and MMT is equal to the time gap between every two launches. Therefore, the availability of a launch system can be written as

$$
A=\frac{\text { MTBM }}{\text { average cycle }}
$$

where, MTBM denotes the time spent for one launch activity and average cycle is the average time gap between two launches including MTBM for a launch system. Please notice that we do not consider the condition of the maintenance of an individual launch vehicle in conflict with a particular launch window. In other words, Eq. (2) is valid for a series, or family, of such launchers during the service period. Here, we use the sample mean $\bar{x}$ to substitute the average cycle. Note that MTBM is a measurement that varies for different space missions. Thus, the factor, sample mean launch cycle $\bar{x}$, is preferred to indicate the level of availability in this case. That is, for the same MTBM, the shorter the $\bar{x}$, the more frequent the launch system is in action and the greater the availability of the launch opportunity.

\subsubsection{Confidence intervals analysis}

As an unbiased estimator of the true mean of the launch cycle, $\bar{x}$ is able to reflect the regular patterns of launch activities as well as the average level of the availability of the launch system. However, this significant statistic parameter fails to illustrate the fluctuation in launch activities. Therefore, in order to supplement the explanation of launch availability, we further derive the confidence interval of the average launch cycle based on the analysis of the launch activity history of space launch systems.

To begin with, a comparison of sample mean of the launch

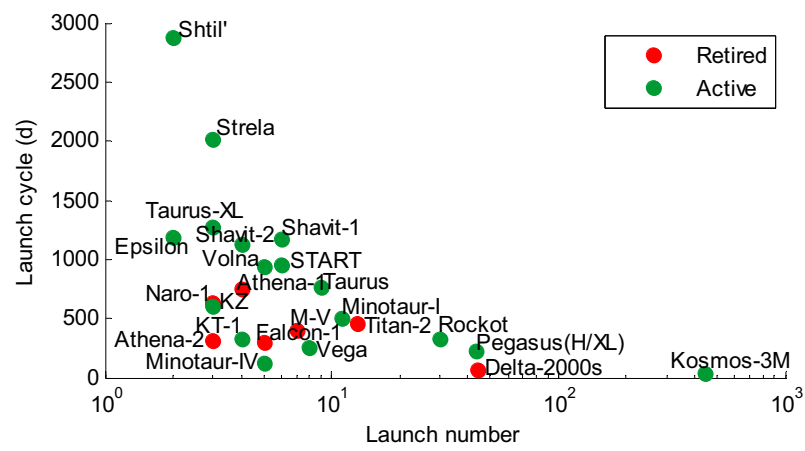

Fig. 2. Total launch times vs. average launch cycle for small launch vehicles.

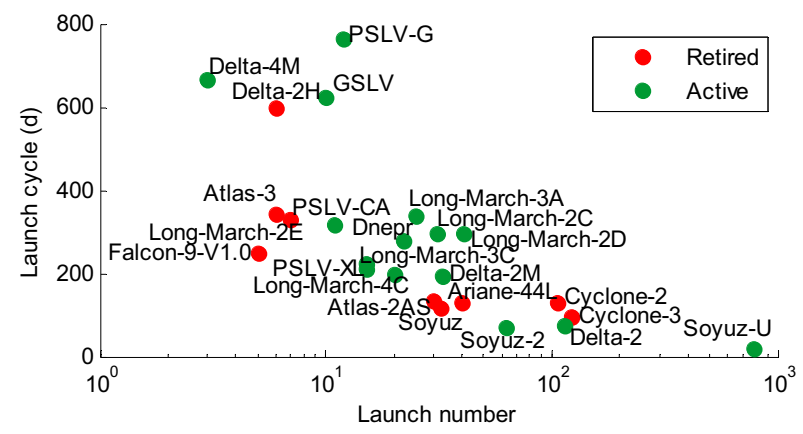

Fig. 3. Total launch times vs. average launch cycle for medium-sized launch vehicles.

cycle and total launch times of different launch systems is presented in Fig. 2 and Fig. 3, with colors differentiating the retired and active systems. As is shown in the graphs, compared with their larger competitors, most of the small launch vehicles have a comparatively longer launch cycle and fewer historical launches. One trend shared by both figures is that, generally, the one with a shorter cycle has more 


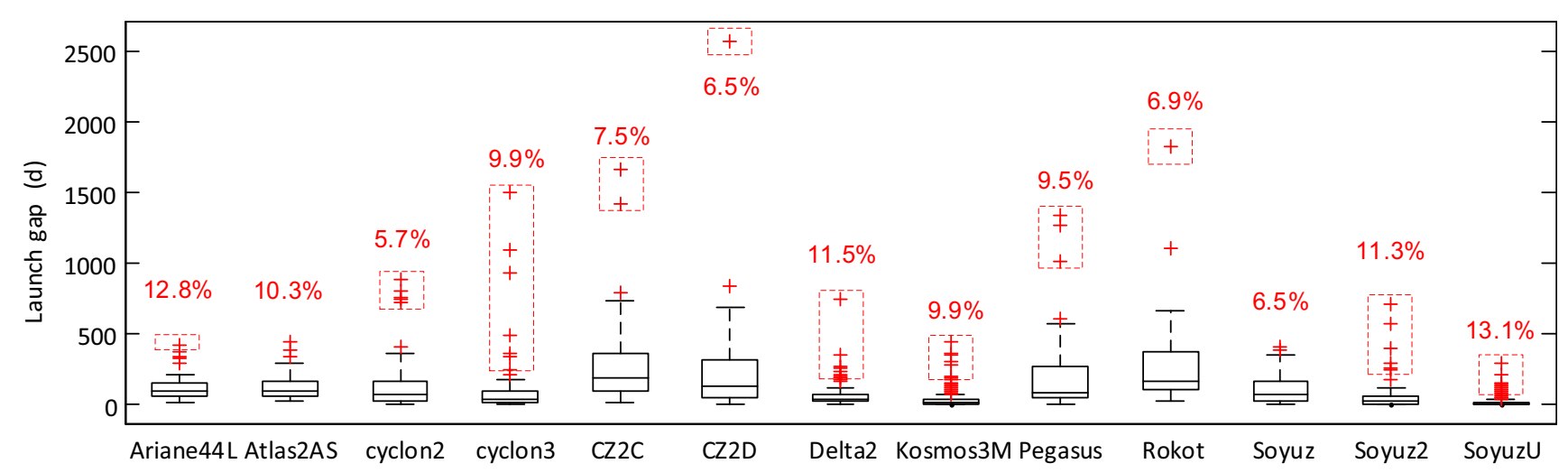

Fig. 4. Box plots show median values (solid horizontal line), lower and upper quartiles ( $Q_{1}$ and $Q_{3}$ at box outlines), lower and upper fences (quartiles $\pm 1.5 \mathrm{IQR}$, whiskers, where IQR $=Q_{3}-Q_{1}$ ), mild outliers and extreme outliers (red plus outside and inside red dashed boxes) and abnormal data rate indicated by red percentages.

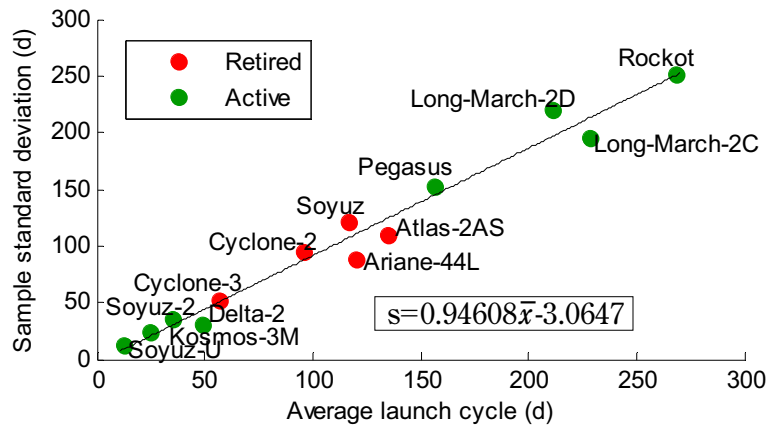

Fig. 5. Launch cycle vs. SSD with regression line by OLS (selected launch systems).

launches (e.g., points located in the right bottom), which denotes a higher level of technical maturity.

For further analysis, we select 13 launch systems from both categories to study the statistical facts in the launch activity record. All of the representatives should have a number of launch records over 20 to be considered a reliable data source. From examination of the fence points and data, each group has points exceeding the upper fence with an abnormal data rate ranging from $5.7 \%$ to $13.1 \%$, where the abnormal data rate is equal to the percentage of outliers in the entire sample (see the red tags in Fig. 4). These extreme outliers (>quartiles + 3IQR) marked with red dashed boxes in Fig. 4 are removed, while mild outliers are kept as a caution. Then, in the scatter plot of the trimmed data, shown in Fig. 5, a positive correlation can be observed with respect to the average value of the launch cycles $\bar{x}$ and the corrected sample standard deviation $s$. Therefore, a linear regression function using the ordinary least squares (OLS) method is applied

$$
s=0.94608 \bar{x}-3.0647, \quad R^{2}=0.9717
$$

where, $R^{2}$ is the coefficient of determination.

In order to check whether or not the regression model proposed fits the data well, a $F$-test is conducted applying a significant level $\alpha=0.01$ to test the null hypothesis for lack of linear fit. Since the $F$ statistic, in this case equaling 377.9223, is greater than the critical $F$ value $F_{1-\alpha}(1, n-2)=$
$F_{0.99}(1,11)=9.65$, the null hypothesis could be rejected; meaning that the linear model proposed fits well.

To further decide the confidence interval for the estimate of the true mean $\mu$, especially the upper limit of the mean representing the longest possible launch cycle, a one-sided test for true mean is adopted. Since the standard deviation $\sigma$ is unknown for the small size samples, Student's $t$-distribution is used as the critical value. Suppose that the test statistic $T=(\bar{x}-\mu) /(s / \sqrt{n})$ is distributed as a Student's $t$-distribution with $n-1 \mathrm{deg}$ of freedom, there is a probability $1-\alpha$ that $T$ could be less than $t_{1-\alpha}(n-1)$

$$
\begin{aligned}
P_{r}\left(T<t_{1-\alpha}(n-1)\right) & =P_{r}\left(\frac{\bar{x}-\mu}{s / \sqrt{n}}<t_{1-\alpha}(n-1)\right) \\
& =(1-\alpha) \times 100 \%
\end{aligned}
$$

where, $n$ is the sample size, and $s$ is the sample standard deviation yield to the regression function (Eq. (3)) for those launch systems with limited launch attempts. Then, the upper confidence limit (UCL) of the mean with a confidence level $(1-\alpha)$ can be calculated using the following equation:

$$
\mathrm{UCL}_{1-\alpha}=\bar{x}+t_{1-\alpha}(n-1) \frac{s}{\sqrt{n}}
$$

The resulting UCL denotes the greatest average value that will occur for a given confidence level and population size. As for the variable launch cycle, this introduces an upper bound that the possible average launch cycle could be shorter than with the possibility of $1-\alpha$.

Therefore, with the average launch cycle $\bar{x}$ and sample standard $s$ as the inputs, the upper confidence limit (UCL) of the mean of launch cycle of each launch system can be obtained (shown in Table 3 ) taking $\alpha$ as 0.05 . The higher the rank, the less maximum potential waiting time for a launch opportunity. From Table 3, we also find that, although in general, launch systems whose average launch cycles are shorter usually have a shorter UCL of mean as well; however, from a local comparison, those with a shorter $\bar{x}$ do not always rank high (e.g., KT-1). As a result, the UCL of the mean for a launch cycle could serve as a supplementary judgment index of launch availability to help with decision- 
Table 3. Ranking of the $\mathrm{UCL}_{0.95}$ of the mean of small launch systems, and medium and intermediate launch systems. (The average launch cycles of 13 selected launch vehicles (shown in Fig. 5) are calculated from data sorted by deleting the extreme outliers, which in this table results in different values from those in Table 1 and Table 2.)

\begin{tabular}{|c|c|c|c|c|c|c|c|}
\hline No. & Small launch system & $\mathrm{UCL}_{0.95}(\mathrm{~d})$ & $\begin{array}{l}\text { Average launch } \\
\text { cycle (d) }\end{array}$ & No. & $\begin{array}{l}\text { Medium and intermediate } \\
\text { launch systems }\end{array}$ & $\mathrm{UCL}_{0.95}(\mathrm{~d})$ & $\begin{array}{c}\text { Average launch } \\
\text { cycle (d) }\end{array}$ \\
\hline 1 & Kosmos 3M & 40 & 35 & 1 & Soyuz U & 14 & 13 \\
\hline 2 & Delta 2000-Series & 81 & 66 & 2 & Soyuz 2 & 43 & 35 \\
\hline 3 & Minotaur IV & 273 & 131 & 3 & Delta 2 & 54 & 49 \\
\hline 4 & Pegasus & 316 & 231 & 4 & Cyclone 3 & 65 & 57 \\
\hline 5 & Vega & 423 & 251 & 5 & Cyclone 2 & 111 & 96 \\
\hline 6 & Rockot & 444 & 322 & 6 & Ariane 44L & 145 & 121 \\
\hline 7 & Falcon 1 & 635 & 302 & 7 & Soyuz & 153 & 116 \\
\hline 8 & Titan 2 & 684 & 460 & 8 & Atlas 2AS & 169 & 135 \\
\hline 9 & $\mathrm{M}-\mathrm{V}$ & 714 & 403 & 9 & Delta $2 \mathrm{M}$ & 248 & 194 \\
\hline 10 & Minotaur I & 780 & 505 & 10 & Long March 2D & 280 & 211 \\
\hline 11 & KT-1 & 858 & 333 & 11 & Long March 2C & 282 & 228 \\
\hline 12 & Taurus & 1258 & 771 & 12 & PSLV-XL & 305 & 212 \\
\hline 13 & Athena 2 & 1619 & 313 & 13 & Long March 3C & 323 & 224 \\
\hline 14 & START & 1815 & 956 & 14 & Atlas 3 & 343 & 343 \\
\hline 15 & Athena 1 & 1931 & 746 & 15 & Dnepr & 374 & 277 \\
\hline 16 & Volna & 1991 & 944 & 16 & Long March 3A & 447 & 337 \\
\hline 17 & Shavit 1 & 2215 & 1166 & 17 & PSLV-CA & 488 & 316 \\
\hline 18 & Shavit 2 & 2920 & 1127 & 18 & Falcon 9 V1.0 & 525 & 250 \\
\hline 19 & $\mathrm{KZ}$ & 3126 & 601 & 19 & GSLV & 990 & 625 \\
\hline 20 & Naro-1 & 3262 & 627 & 20 & PSLV-G & 1157 & 764 \\
\hline 21 & Taurus XL & 6621 & 1270 & 21 & Delta 4M & 3471 & 667 \\
\hline 22 & Strela & 10518 & 2016 & & & & \\
\hline
\end{tabular}

making when there are various options yet the performance levels are very close to each other.

\subsection{Reliability analysis}

The possibility of the future success for the next launch is of great importance in performance assessment. Let $s$ denote the number of successful launches observed in a random launch record of $t$ attempts. Assume that we know nothing before the launch of a given vehicle, thus the proportion $p$ of successful launches is uniformly distributed over the inter$\operatorname{val}(0,1),{ }^{17)}$

$$
g(p)= \begin{cases}1 & 0 \leq p \leq 1 \\ 0 & \text { elsewhere }\end{cases}
$$

where, the probability density function $g(p)$ is a prior distribution. For a given $p, s$ will have a binomial distribution given by

$$
f(s \mid p)=\left(\begin{array}{l}
t \\
s
\end{array}\right) p^{s}(1-p)^{t-s} \quad s=0,1, \ldots, t
$$

Then, we can have the density function of the distribution for the future fraction of successful launches

$$
\begin{aligned}
f(p \mid s)= & \frac{f(s \mid p) g(p)}{\int_{0}^{1} f\left(s \mid p^{\prime}\right) g\left(p^{\prime}\right) d p^{\prime}} \\
= & \frac{\Gamma(s+1) \Gamma(t-s+1)}{\Gamma(t+2)} p^{s}(1-p)^{t-s} \\
& 0 \leq p \leq 1
\end{aligned}
$$

which is the beta density function: beta $(s+1, t-s+1)$.

Thus, the Bayes estimate of $p$ is the mean of this posterior density function; namely,

$$
\int_{0}^{1} p^{\prime} f\left(p^{\prime} \mid s\right) d p^{\prime}=\frac{s+1}{t+2}
$$

According to the study by Howard, ${ }^{18)}$ the best estimate of probability of success in the next launch for a given launch vehicle is the mean of the posterior distribution for future frequency of launch success. Therefore, the predicted reliability of active representative launch vehicles can be calculated using Eq. (9). The results are illustrated in Table 4. Figures 6 and 7 also demonstrate the results with the total launch times and colors representing the average launch cycle of each launch vehicle. Note that some points coinciding with others for closing value can still be differentiated by their performance in average launch cycle (e.g., cycle $_{(\text {Kuaizhou })}=$ $601 \mathrm{~d}, \quad \operatorname{cycle}_{(\text {Strela) }}=2,016 \mathrm{~d}, \quad$ cycle $_{(\text {Epsilon })}=1,193 \mathrm{~d}$ and $\left.\operatorname{cycle}_{\left(\text {Shtil' }^{\prime}\right)}=2,880 \mathrm{~d}\right)$.

Compared to the small launch vehicles shown in Fig. 6, the medium and intermediate launch vehicles in Fig. 7 have better performance in reliability, experience and availability with higher predicted reliability, more total launch times, and shorter average launch cycles, which can also be found in Table 4. Moreover, points with a warm color in Fig. 7 tend to converge in the upper part of the graph (i.e., predicted reliability $\geq 90 \%$ ), with more launches than green points. This indicates a close relationship between abundant experience, higher availability and better reliability. As for small launch vehicles, there is no such obvious trend shown in the graph. However, when only considering those with more than 10 launches, a similar conclusion will be obtained that more launch experience realizes progress in technique maturity, especially in terms of predicted reliability.

Note that the predicted reliability of some launch vehicles may differ significantly from the success rate achieved (e.g., 
Trans. Japan Soc. Aero. Space Sci., Vol. 61, No. 2, 2018

Table 4. Comparison between success rate and predicted reliability.

\begin{tabular}{|c|c|c|c|}
\hline $\begin{array}{l}\text { Launch } \\
\text { vehicle }\end{array}$ & $\begin{array}{c}\text { Launch } \\
\text { success/Total } \\
\text { launches }\end{array}$ & $\begin{array}{l}\text { Success } \\
\text { rate }(\%)\end{array}$ & $\begin{array}{l}\text { (Predicted reliability, } \\
\text { standard deviation) (\%) }\end{array}$ \\
\hline \multicolumn{4}{|l|}{ Small } \\
\hline Epsilon & $2 / 2$ & 100.00 & $(75.00,19.36)$ \\
\hline Kosmos 3M & $424 / 426$ & 95.07 & $(94.87,1.04)$ \\
\hline KT-1 & $2 / 4$ & 50.00 & $(50.00,18.90)$ \\
\hline Kuaizhou/KZ & $3 / 3$ & 100.00 & $(80.00,16.33)$ \\
\hline Long March 11 & $1 / 1$ & 100.00 & $(66.67,23.57)$ \\
\hline Long March 6 & $1 / 1$ & 100.00 & $(66.67,23.57)$ \\
\hline Minotaur I & $11 / 11$ & 100.00 & $(92.31,7.12)$ \\
\hline Minotaur IV & $5 / 5$ & 100.00 & $(85.71,12.37)$ \\
\hline Pegasus XL & $29 / 33$ & 87.88 & $(85.71,5.83)$ \\
\hline Pegasus & $38 / 43$ & 88.37 & $(86.67,5.01)$ \\
\hline Rockot & $27 / 30$ & 90.00 & $(87.50,5.76)$ \\
\hline Shavit 1 & $4 / 6$ & 66.67 & $(62.50,16.14)$ \\
\hline Shavit 2 & $4 / 4$ & 100.00 & $(83.33,14.09)$ \\
\hline Shtil 1 & $2 / 2$ & 100.00 & $(75.00,19.36)$ \\
\hline START & $6 / 6$ & 100.00 & $(87.50,11.02)$ \\
\hline Strela & $3 / 3$ & 100.00 & $(80.00,16.33)$ \\
\hline Taurus XL & $1 / 3$ & 33.33 & $(40.00,20.00)$ \\
\hline Taurus & $6 / 9$ & 66.67 & $(63.64,13.89)$ \\
\hline Vega & $8 / 8$ & 100.00 & $(90.00,9.05)$ \\
\hline Volna & $2 / 5$ & 40.00 & $(42.86,17.50)$ \\
\hline \multicolumn{4}{|l|}{ Medium } \\
\hline Delta 2 & $112 / 114$ & 98.39 & $(97.41,1.43)$ \\
\hline Delta $2 \mathrm{M}$ & $33 / 33$ & 100.00 & $(97.14,2.78)$ \\
\hline Delta 4M & $3 / 3$ & 100.00 & $(80.00,16.33)$ \\
\hline Dnepr & $21 / 22$ & 95.45 & $(91.67,5.53)$ \\
\hline GSLV & $5 / 10$ & 50.00 & $(50.00,13.87)$ \\
\hline Long March 2C & $40 / 41$ & 97.56 & $(95.35,3.17)$ \\
\hline Long March 2D & $31 / 31$ & 100.00 & $(96.97,2.94)$ \\
\hline Long March 3A & $25 / 25$ & 100.00 & $(96.30,3.57)$ \\
\hline Long March 3C & $15 / 15$ & 100.00 & $(94.12,5.55)$ \\
\hline Long March 4C & $19 / 20$ & 95.00 & $(90.91,5.99)$ \\
\hline PSLV-CA & $11 / 11$ & 100.00 & $(92.31,7.12)$ \\
\hline PSLV-G & $10 / 12$ & 83.33 & $(78.57,10.59)$ \\
\hline PSLV-XL & $15 / 15$ & 100.00 & $(94.12,5.55)$ \\
\hline Soyuz 2 & $58 / 63$ & 91.94 & $(90.77,3.58)$ \\
\hline Soyuz U & $764 / 785$ & 97.32 & $(97.20,0.59)$ \\
\hline
\end{tabular}

Long March 11, Long March 6 and Taurus XL). This is due to prior knowledge and lack of launch data. With the accumulation of launch experience, the impact from prior knowledge is reduced while the number of launches has a greater impact, thereby reducing the difference between predicted reliability and current success rate. To accurately evaluate launch systems with limited launch data, efforts could be made to find other prior distributions to make full use of all available information.

\section{Aggregated Preferences Value Analysis}

Although the updated launch vehicle database provides practical indices to evaluate launch opportunities, it is less straightforward in providing reliable views standing for all concerned attributes by a series of values rather than a standard unit. A more intuitive and understandable method to evaluate the comprehensive capabilities of various launch systems is developed using decision-maker (DM) preferences. In this part, the preference types of different attributes are first introduced. Then, the translation procedure is imple-

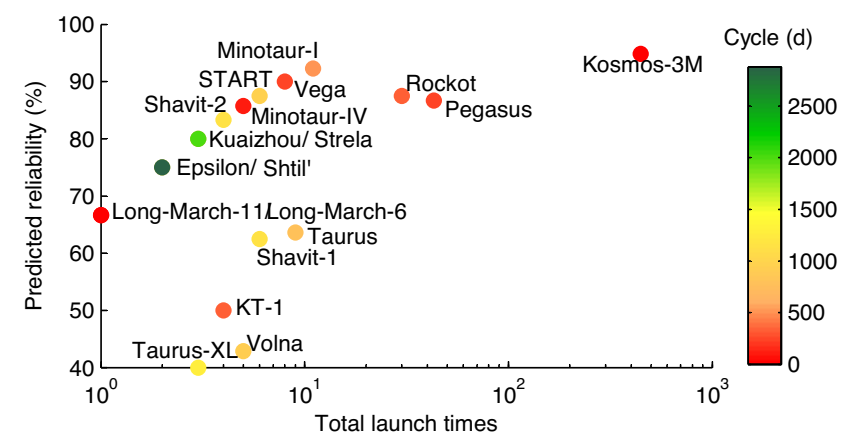

Fig. 6. Scatter plot of predicted reliability of small launch vehicles.

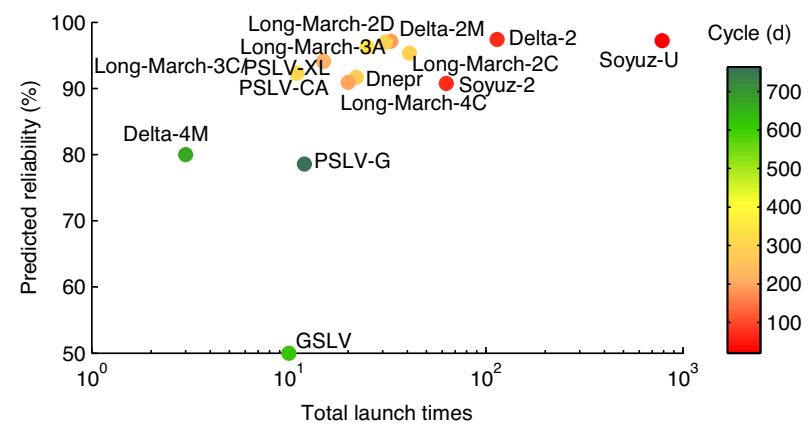

Fig. 7. Scatter plot of predicted reliability of medium launch vehicles.

mented to transform essential knowledge and concerns from the DM preferences into separate quantified ranges. Finally, an aggregated preferences value function is established to integrate all of the attributes into a single metric. With the aggregated preferences value, the promising launch opportunity can be selected while taking designed objectives into account.

\subsection{Classifications of objectives}

As is described in the theory of physical programming, ${ }^{19)}$ the types of different criteria depend on the sharpness of the preference. In this study, there are two types of preferences: 1) Type-1: smaller is better and 2) Type-2: larger is better. For criterion $i$, there are $N$ ranges to differentiate the levels of desirability towards all of the $M$ criteria. The physical values of the criterion $i$ at the boundaries of the ranges $f_{i}^{k}(i=1, \ldots, M ; k=0,1, \ldots, N)$ are used to quantify the preferences in an ascending order, with the highest preference value for the first and lowest for the last. In an actual case, these standards are set mostly by the experience and knowledge of the experts. An example of designed desirability $(N=5)$ is shown in Table 5 .

\subsection{Aggregated preference function}

The mathematic formulation of the preference function is established in order to quantify the different criteria in a relatively fair way. Aiming at this goal, the same set of images $\alpha_{k}$ is implemented for preference function construction in each value range respectively with a piecewise exponential function. ${ }^{20)}$ Those $\alpha_{k}$ at boundaries $f_{i}^{k}$ can be expressed as

$$
\alpha_{k}= \begin{cases}0, & k=0 \\ M^{k-1} \cdot \alpha_{i n i}, & 1 \leq k \leq N\end{cases}
$$


Table 5. Preference order and their attribution of Type- 1 and Type-2.

\begin{tabular}{clll}
\hline Level & \multicolumn{1}{c}{ Description } & Type-1 & Type-2 \\
\hline A & Highly desirable & $f_{i}^{0}<f_{i} \leq f_{i}^{1}$ & $f_{i}^{1} \leq f_{i}<f_{i}^{0}$ \\
B & Desirable & $f_{i}^{1}<f_{i} \leq f_{i}^{2}$ & $f_{i}^{2} \leq f_{i}<f_{i}^{1}$ \\
C & Tolerable & $f_{i}^{2}<f_{i} \leq f_{i}^{3}$ & $f_{i}^{3} \leq f_{i}<f_{i}^{2}$ \\
D & Undesirable & $f_{i}^{3}<f_{i} \leq f_{i}^{4}$ & $f_{i}^{4} \leq f_{i}<f_{i}^{3}$ \\
E & Highly undesirable & $f_{i}^{4}<f_{i} \leq f_{i}^{5}$ & $f_{i}^{5} \leq f_{i}<f_{i}^{4}$ \\
\hline
\end{tabular}

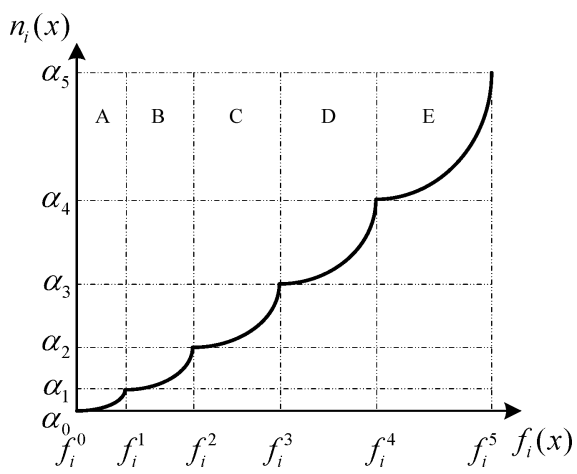

Fig. 8. Preference function Type-1 with $N=5 .^{14)}$

where $\alpha_{i n i}$ is a constant that is used to compare the relative preferences of different systems. Thus, image $\alpha_{i n i}$ can be chosen randomly from any small value as its absolute value does not make a difference. Here $\alpha_{i n i}=0.02$ for the rest of this paper.

(1) Type-1: the smaller the better

Without loss of generality, we define the preference function with $n$ order in the range $k$ (e.g. $f_{i}^{k-1}<f_{i}(x) \leq f_{i}^{k}$ ) as

$$
p_{k}(x)=\alpha_{k-1}+\beta_{k}\left|1-e^{-n\left(\frac{f_{i}(x)-f_{i}^{k-1}}{f_{i}^{k}-f_{i}^{k-1}}\right)}\right|
$$

For the following examples, $n$ is set to be -2 . Since $p_{k}\left(f_{i}^{k}\right)=\alpha_{k}$, the parameter $\beta_{k}$ can be obtained.

$$
\beta_{k}=\frac{\alpha_{k}-\alpha_{k-1}}{\left|1-e^{-n}\right|}
$$

As a result, for criterion $i$, the Type- 1 preference function can be expressed as

$$
n_{i}^{1}(x)= \begin{cases}0, & f_{i}(x)<f_{i}^{0} \\ p_{k}(x), & f_{i}^{k-1} \leq f_{i}(x) \leq f_{i}^{k} \\ p_{N}(x), & f_{i}(x)>f_{i}^{N}\end{cases}
$$

with $1 \leq k \leq N$ and $p_{N}(x)=\alpha_{N}$.

(2) Type-2: the larger the better

Similarly, the preference function for criterion $i$ of type-2 can be defined as

$$
n_{i}^{2}(x)= \begin{cases}0, & f_{i}(x)>f_{i}^{0} \\ p_{k}(x), & f_{i}^{k} \leq f_{i}(x) \leq f_{i}^{k-1} \\ p_{N}(x), & f_{i}(x)<f_{i}^{N}\end{cases}
$$

Examples of these two type preference functions are given in Figs. 8 and 9.

As soon as the preference function in each value range is defined, we use an aggregated preference function $V(x)$ to integrate the preference value of all the criteria.

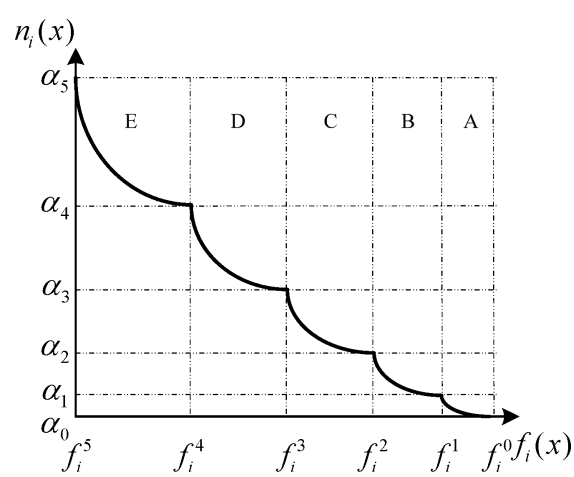

Fig. 9. Preference function Type-2 with $N=5 .{ }^{14)}$

Table 6. Preferences of four criteria for launch vehicles.

\begin{tabular}{lcrccccr}
\hline \multicolumn{1}{c}{ Criterion } & Type & \multicolumn{1}{c}{$f_{i}^{0}$} & $f_{i}^{1}$ & $f_{i}^{2}$ & $f_{i}^{3}$ & $f_{i}^{4}$ & $f_{i}^{5}$ \\
\hline Unit cost (FY2010\$K/kg) & 1 & 1 & 15 & 25 & 35 & 50 & 500 \\
Predicted reliability (\%) & 2 & 100 & 95 & 90 & 80 & 70 & 0 \\
Total launches & 2 & 1000 & 101 & 51 & 21 & 5 & 0 \\
Availability & 2 & 1 & $1 / 3$ & $1 / 9$ & $1 / 18$ & $1 / 36$ & $1 / 96$ \\
\hline
\end{tabular}

$$
V(x)=\sum_{i=1}^{M} n_{i}(x)
$$

Once the aggregated preference function is constructed, the original multi-criteria optimization problem in this decision-making process can be transformed into a single criterion optimization problem taking multiple constraints into account.

$$
\begin{array}{lll} 
& \multicolumn{2}{c}{\min _{\boldsymbol{x}} J(\boldsymbol{x})=\min V(\boldsymbol{x})} \\
& \boldsymbol{h}_{\boldsymbol{I}}(\boldsymbol{x}) \leq \boldsymbol{h}_{\boldsymbol{I}} & \text { for inequal constraints } \\
\text { s.t. } & \boldsymbol{h}_{\boldsymbol{E}}(\boldsymbol{x}) \leq \boldsymbol{h}_{\boldsymbol{E}} & \text { for equal constraints } \\
& \boldsymbol{x}_{\min } \leq \boldsymbol{x} \leq \boldsymbol{x}_{\max } & \text { for designed variables }
\end{array}
$$

In this study, $\boldsymbol{x}$ represents the preference variables of existing launch systems defining the launch opportunity.

Currently, four indices (i.e., launch cost, predicted reliability, total launch times and availability) are adopted to evaluate the launch opportunity, with the first one categorized into Type- 1 and the others into Type-2. The preferences of each criterion based on expert opinion are described in Table 6. Since the demand is high efficiency, we set MTBM $=30 \mathrm{~d}$ in the definition of availability so that this attribute becomes $100 \%$ if the launch system is operable within one month.

Applying the method developed, both categories of current launch systems are ranked by their preference values. The results are shown in Table 7 and Table 8. For each category, a comparison of the performance of the top six systems is displayed using radar charts in Figs. 10 and 11. By investigating the ranks of Pegasus (fifth with one $\mathrm{B}$, two $\mathrm{C}$ and one D) and Minotaur IV (sixth with one A, one B, one $\mathrm{C}$ and one E) in Fig. 10, it is indicated that balanced development on all of the attributes leads to a higher aggregated preference value. Additionally, since the evaluating images $\alpha_{k}$ are the same for both the small and medium and intermediate launch systems, the graphical quantitative comparison con- 
Trans. Japan Soc. Aero. Space Sci., Vol. 61, No. 2, 2018

Table 7. Preference ranking of active small launch systems with preference ranges.

\begin{tabular}{|c|c|c|c|c|c|c|c|c|c|}
\hline \multirow[b]{2}{*}{ Vehicle } & \multicolumn{2}{|c|}{ LEO cost $(\mathrm{FY} 2010 \$ \mathrm{~K})$} & \multicolumn{2}{|c|}{ Predicted reliability (\%) } & \multicolumn{2}{|c|}{ Total launches } & \multicolumn{2}{|c|}{ Availability } & \multirow[b]{2}{*}{$V(x)$} \\
\hline & $n_{1}^{1}(x)$ & Range & $n_{2}^{2}(x)$ & Range & $n_{3}^{2}(x)$ & Range & $n_{4}^{2}(x)$ & Range & \\
\hline Kosmos 3M & 0.0184 & $\mathrm{~A}$ & 0.0236 & $\mathrm{~B}$ & 0.0164 & $\mathrm{~A}$ & 0.0083 & $\mathrm{~A}$ & 0.0667 \\
\hline Rockot & 0.0163 & A & 0.1892 & $\mathrm{C}$ & 0.2891 & $\mathrm{C}$ & 0.2116 & $\mathrm{C}$ & 0.7061 \\
\hline Vega & 0.0498 & B & 0.080 & $\mathrm{C}$ & 1.2116 & $\mathrm{D}$ & 0.0793 & $\mathrm{~B}$ & 1.4207 \\
\hline Pegasus & 1.0532 & $\mathrm{D}$ & 0.2151 & $\mathrm{C}$ & 0.1947 & $\mathrm{C}$ & 0.0783 & $\mathrm{~B}$ & 1.5413 \\
\hline Pegasus XL & 0.9636 & $\mathrm{D}$ & 0.2398 & $\mathrm{C}$ & 0.2740 & $\mathrm{C}$ & 0.0795 & B & 1.5568 \\
\hline Minotaur IV & 0.0191 & A & 0.2398 & $\mathrm{C}$ & 1.2800 & $\mathrm{E}$ & 0.0621 & $\mathrm{~B}$ & 1.6011 \\
\hline Minotaur I & 0.4455 & $\mathrm{D}$ & 0.0658 & B & 1.1121 & $\mathrm{D}$ & 0.3143 & $\mathrm{C}$ & 1.9378 \\
\hline START & 0.0612 & B & 0.1892 & $\mathrm{C}$ & 1.2600 & $\mathrm{D}$ & 1.2354 & $\mathrm{D}$ & 2.7458 \\
\hline Taurus & 0.0569 & B & 2.0183 & $\mathrm{E}$ & 1.1825 & $\mathrm{D}$ & 1.0955 & $\mathrm{D}$ & 4.3533 \\
\hline $\mathrm{KZ}$ & 0.0154 & A & 0.3200 & $\mathrm{D}$ & 3.7255 & $\mathrm{E}$ & 0.6905 & $\mathrm{D}$ & 4.7515 \\
\hline Volna & 0.0177 & A & 3.6761 & $\mathrm{E}$ & 1.2800 & $\mathrm{E}$ & 1.2297 & $\mathrm{D}$ & 6.2035 \\
\hline Shavit 1 & 1.4446 & $\mathrm{E}$ & 2.1366 & $\mathrm{E}$ & 1.2600 & $\mathrm{D}$ & 2.2154 & $\mathrm{E}$ & 7.0567 \\
\hline Epsilon & 0.2849 & $\mathrm{C}$ & 1.0218 & $\mathrm{D}$ & 4.3834 & $\mathrm{E}$ & 2.4412 & $\mathrm{E}$ & 8.1313 \\
\hline Strela & 0.0160 & A & 0.3200 & $\mathrm{D}$ & 3.7255 & $\mathrm{E}$ & 4.7158 & $\mathrm{E}$ & 8.7773 \\
\hline Shtil 1 & 0.0291 & B & 1.0218 & $\mathrm{D}$ & 4.3834 & $\mathrm{E}$ & 5.1200 & $\mathrm{E}$ & 10.554 \\
\hline Taurus XL & 0.2197 & $\mathrm{C}$ & 3.8364 & $\mathrm{E}$ & 3.7255 & $\mathrm{E}$ & 2.9695 & $\mathrm{E}$ & 10.751 \\
\hline
\end{tabular}

Table 8. Preference ranking of active medium-sized launch systems with preference ranges.

\begin{tabular}{|c|c|c|c|c|c|c|c|c|c|}
\hline \multirow{2}{*}{ Vehicle } & \multicolumn{2}{|c|}{ LEO cost (FY2010\$K) } & \multicolumn{2}{|c|}{ Predicted reliability (\%) } & \multicolumn{2}{|c|}{ Total launches } & \multicolumn{2}{|c|}{ Availability } & \multirow{2}{*}{$V(x)$} \\
\hline & $n_{1}^{1}(x)$ & Range & $n_{2}^{2}(x)$ & Range & $n_{3}^{2}(x)$ & Range & $n_{4}^{2}(x)$ & Range & \\
\hline Soyuz U & 0.0144 & A & 0.0156 & A & 0.0088 & A & 0 & A & 0.0387 \\
\hline Delta 2 & 0.0198 & A & 0.0149 & A & 0.0199 & A & 0.0192 & A & 0.0738 \\
\hline Soyuz 2 & 0.0132 & A & 0.0766 & $\mathrm{~B}$ & 0.0742 & B & 0.0191 & A & 0.1831 \\
\hline Long March 2C & 0.0163 & A & 0.0195 & A & 0.2151 & $\mathrm{C}$ & 0.1569 & $\mathrm{C}$ & 0.4078 \\
\hline Delta $2 \mathrm{M}$ & 0.0600 & $\mathrm{~B}$ & 0.0158 & A & 0.2740 & $\mathrm{C}$ & 0.0755 & $\mathrm{~B}$ & 0.4252 \\
\hline Long March 2D & 0.0116 & A & 0.0162 & A & 0.2844 & $\mathrm{C}$ & 0.1638 & $\mathrm{C}$ & 0.4761 \\
\hline Dnepr & 0.0093 & A & 0.0711 & B & 0.3174 & $\mathrm{C}$ & 0.1067 & $\mathrm{C}$ & 0.5045 \\
\hline Long March 3A & 0.0166 & A & 0.0179 & A & 0.3085 & $\mathrm{C}$ & 0.2321 & $\mathrm{C}$ & 0.5750 \\
\hline Long March 4C & 0.0179 & A & 0.0759 & B & 0.4505 & $\mathrm{D}$ & 0.0760 & B & 0.6202 \\
\hline PSLV-XL & 0.0106 & A & 0.0406 & B & 0.9058 & $\mathrm{D}$ & 0.0770 & B & 1.0341 \\
\hline Long March 3C & 0.0178 & A & 0.0406 & $\mathrm{~B}$ & 0.9058 & $\mathrm{D}$ & 0.0778 & $\mathrm{~B}$ & 1.0421 \\
\hline PSLV-CA & 0.0136 & A & 0.0658 & $\mathrm{~B}$ & 1.1122 & $\mathrm{D}$ & 0.2023 & $\mathrm{C}$ & 1.3938 \\
\hline PSLV-G & 0.0108 & A & 0.5959 & $\mathrm{D}$ & 1.0698 & $\mathrm{D}$ & 1.0870 & $\mathrm{D}$ & 2.7635 \\
\hline Delta 4M & 0.0165 & A & 0.3200 & $\mathrm{D}$ & 3.7255 & $\mathrm{E}$ & 0.9119 & $\mathrm{D}$ & 4.9739 \\
\hline GSLV & 0.0155 & A & 3.2131 & $\mathrm{E}$ & 1.1495 & $\mathrm{D}$ & 0.7846 & $\mathrm{D}$ & 5.1628 \\
\hline
\end{tabular}

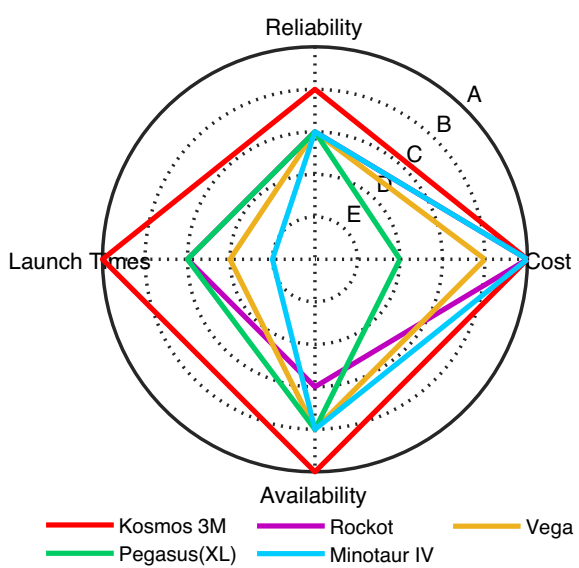

Fig. 10. Comparison of preference towards active small launch systems.

cludes that the overall performance of the latter group is better than the smaller competitors.

For example, the average amount of unit LEO cost of the medium group is only $42.40 \%$ of that of small systems, while the average total launch time of the medium-sized systems is 2.31 times that of the other group.

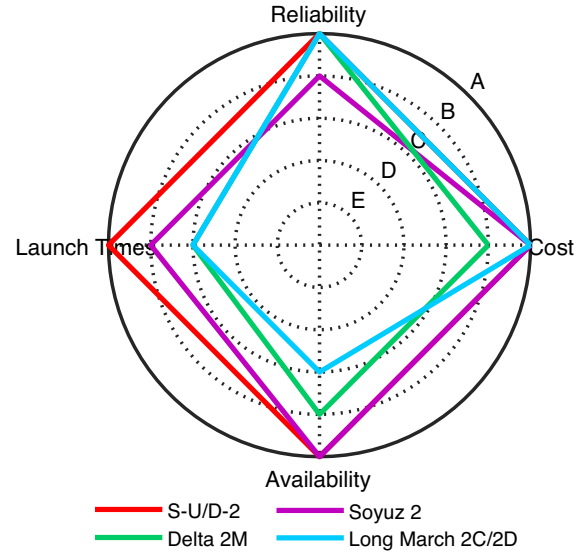

Fig. 11. Comparison of preference towards active medium and intermediate launch systems (S-U denotes Soyuz U and D-2 denotes Delta 2, which rank 1st and 2nd place, respectively, with coinciding lines in the chart).

\section{Case Study}

In order to demonstrate the capability of the method proposed, two classical launch cases of small satellites are re- 
Table 9. Comparison of the potential launch vehicles for the Flock 3p mission.

\begin{tabular}{llccc}
\hline \multirow{2}{*}{ Mission } & \multicolumn{1}{c}{$\begin{array}{c}\text { Expected } \\
\text { launch vehicle }\end{array}$} & $\begin{array}{c}\text { Total cost } \\
(\mathrm{FY} 2010 \$ \mathrm{~K})\end{array}$ & $\begin{array}{c}\text { Predicted } \\
\text { reliability } \\
(\%)\end{array}$ & $V(x)$ \\
\hline Flock 3p & PSLV-XL & 2192.08 & 94.12 & 1.0341 \\
\hline Small & Kosmos 3M & 5004.56 & 94.87 & 0.0667 \\
& Rockot & 3929.20 & 87.50 & 0.7061 \\
& Vega & 7362.08 & 90.00 & 1.4207 \\
& Pegasus XL & 17164.40 & 85.71 & 1.5413 \\
& Minotaur IV & 5500.88 & 85.71 & 1.6011 \\
\hline \multirow{2}{*}{ Medium } & Soyuz U & 3226.08 & 97.20 & 0.0387 \\
& Delta 2 & 6038.56 & 97.41 & 0.0738 \\
& Soyuz 2 & 2853.84 & 90.77 & 0.1831 \\
& Long March 2C & 3929.20 & 95.35 & 0.4078 \\
& Delta 2M & 7982.48 & 97.14 & 0.4252 \\
\hline
\end{tabular}

Table 10. Comparison of the potential launch vehicles for the Formosat-2 mission.

\begin{tabular}{llccc}
\hline \multirow{2}{*}{ Mission } & \multicolumn{1}{c}{$\begin{array}{c}\text { Expected } \\
\text { launch vehicle }\end{array}$} & $\begin{array}{c}\text { Total cost } \\
(\mathrm{FY2010 \$ K)})\end{array}$ & $\begin{array}{c}\text { Predicted } \\
\text { reliability } \\
(\%)\end{array}$ & $V(x)$ \\
\hline Formosat-2 & Taurus-XL & 12380.80 & 40.00 & 10.751 \\
\hline Small & Kosmos 3M & 9244.40 & 94.87 & 0.0667 \\
& Rockot & 7258.00 & 87.50 & 0.7061 \\
& Vega & 13599.20 & 90.00 & 1.4207 \\
& Minotaur IV & 10161.20 & 85.71 & 1.6011 \\
& Taurus & 14363.20 & 63.64 & 4.3533 \\
\hline \multirow{2}{*}{ Medium } & Soyuz U & 5959.20 & 97.20 & 0.0387 \\
& Delta 2 & 11154.40 & 97.41 & 0.0738 \\
& Soyuz 2 & 5271.60 & 90.77 & 0.1831 \\
& Long March 2C & 7258.00 & 95.35 & 0.4078 \\
& Delta 2M & 14745.20 & 97.14 & 0.4252 \\
\hline
\end{tabular}

viewed with their launch vehicles reconsidered by the aggregated preference value function. The only constraint in Eq. (15) considered is the LEO payload capacity. The comparison is shown in Table 9 and Table 10.

The first case is a well-known constellation launch mission, Flock 3p, consisting of 88 cubesats, each weighing $4.7 \mathrm{~kg} .{ }^{21)}$ This constellation was launched by PSLV-XL on 15 February 2017. As is shown in Table 9, we can clearly notice that the outstanding advantage of PSLV-XL is the low cost, which might be the driving factor for Planet Labs to choose it. Simultaneously, one factor that cannot be ignored is its unsatisfactory performance in availability, which puts a negative impact on PSLV-XL's aggregated preference ranking (shown in Table 8). Since this was not an emergency mission, the company's decision leaned towards the cost characteristic. Generally, the Soyuz U is recommended by the preference model for this launch mission in terms of its comprehensive capability.

As is shown in Table 10, the Taiwanese small satellite Formosat-2, ${ }^{22}$ launched using the Taurus XL in 2004, is demonstrated as the second case. Through preference analysis, this launch strategy cannot be considered a sensible one, since none of the attributes of the Taurus XL is superior. By comparing the aggregated preference value of active launch vehicles, Kosmos 3M, Rockot, Soyuz, Delta 2 and Long
March 2C are the top five candidates with convincing performance in such characteristics as cost, reliability, availability, and so on. With any of these potential candidates as a replacement of the launch vehicle of satellite Formosat-2, financial cost as well as potential waiting time could have been saved for launch operation and preparation. However, one condition that cannot be ignored is that political and other non-performance reasons also influence the decisionmaking process, which might account for the final choice of Formosat-2, yet does not fall within the scope of this paper.

\section{Conclusions}

The miniaturization of satellites makes it easier to obtain access to space, yet problems still exist in finding the best launch opportunity for entering space, and this is constrained by concrete or abstract considerations. The aim of this paper was to identify, quantify and evaluate the key concerns of small satellite launch opportunities using an aggregated preference value model applied from the perspective of the user. Such a task could be an initial point of exploration toward the decision-making process when studying small satellite launch opportunities.

Based on our previous research, we updated a launch system capability database that extracts main attributes in two categories: small and medium and intermediate launch vehicles. Subsequently, the definition of the availability of launch opportunity was identified as well as the predicted reliability that allows for further assessment of launch capabilities. This analysis has shown a positive relation between the larger total number of launch attempts and small average launch cycle, as well as greater availability towards launch opportunities. Finally, an aggregated preference value model was proposed to integrate and evaluate the key user-defined objectives of the classified launch vehicle. Our results show that a higher preference tendency exists towards launch systems with balanced development in all aspects (e.g., cost, total launch times, reliability and availability). All in all, there still exists a big opportunity for dedicated launchers to develop technologies and to catch up with the larger competitors in the global market.

This method allows launch opportunities to be estimated without an interdisciplinary trade-off and brings in a simple but concrete index for decision-making. Moreover, the aggregated preference value method developed here provides not only an approach for users to choose from different options, but also a chance for launchers to re-examine their positioning in the demanding global market. Therefore, the method proposed can help select an appropriate launch opportunity for a given mission and obtain a better understanding of the current small satellite launch market.

\section{Acknowledgments}

The authors gratefully thank the China Scholarship Council (CSC) for financial support of this project. 


\section{References}

1) Buchen, E.: Small Satellite Market Observations, Proceedings of the AIAA/USU Conference on Small Satellites, Logan, UT, USA, Aug. 2015 .

2) Lim, B.: Small Satellite Developments, 2016 IEEE International Geoscience and Remote Sensing Symposium (IGARSS), 2016, pp. 5543-5545.

3) Niederstrasser, C. and Frick, W.: Small Launch Vehicles - A 2015 State of the Industry Survey, AIAA/USU Conference on Small Satellites, Logan, UT, USA, Technical Session VII: Opportunities, Trends and Initiatives, 2015.

4) Crisp, N., Smith, K., and Hollingsworth, P.: Small Satellite Launch to LEO: A Review of Current and Future Launch Systems, Trans. JSASS Aerospace Technology Japan, 12, ists29 (2014), Tf_39-Tf_47.

5) Doncaster, B., Shulman, J., Bradford, J., and Olds, J.: Spaceworks' 2016 Nano/Microsatellite Market Forcast, the 30th Annual AIAA/ USU Conference on Small Satellites, Logan, UT, USA, 2016, SSC16-II-01.

6) Borah, R.: In India, the Modi Juggernaut Rolls On, Global Asia: J. East Asia Foundation, 12 (2017), p. 98.

7) Union of Concerned Scientists: UCS Satellite Database, http: //www.ucsusa.org/nuclear-weapons/space-weapons/satellite-database\#. WfEKnWhL9PZ (accessed January 31, 2017).

8) Hopkins, J., Jr. and Hopkins, J.: International Reference Guide to Space Launch Systems, Fourth Ed., American Institute of Aeronautics and Astronautics, Reston, VA, 2004.

9) Wertz, J. R., Everett, D. F., and Puschell, J. J.: Space Mission Engineering: the New SMAD, Microcosm Press, Hawthorne, CA, 2011.

10) Encyclopedia Astronautica, Available from: http://www.astronautix. com/ (accessed March 14, 2017).

11) Gunter's Space Page - Information on Spaceflight, Launch Vehicles and Satellites, Available from: http://space.skyrocket.de/ (accessed
March 14, 2017)

12) Space Launch Report, Available from: http://spacelaunchreport.com/ (accessed March 14, 2017).

13) Boone, T. R., IV and Miller, D. P.: Capability and Cost-Effectiveness of Launch Vehicles, New Space, 4 (2016), pp. 168-189.

14) Xu, Q., Zhang, M., Hao, Z., Hollingsworth, P., and Smith, K.: Small Satellite Launch Opportunities: Statistical Analysis and Trend Forecast, the 67th International Aeronautical Congress, Guadalajara, Mexico, 2016, IAC-16,B4,5,9,x35391.

15) Davidson, J.: The Reliability of Mechanical Systems, Mechanical Engineering Publications, London, 1988, pp. 13-15.

16) Jones, W. D., Jr.: Glossary Defense Acquisition Acronyms and Terms, The Department of Defense Systems Management College, Fort Belvoir, VA, 1991.

17) Scheaffer, R. and McClave, J.: Probability and Statistics for Engineers, 3rd Ed., Thomson Information/Publishing Group, Boston, 1990.

18) Howard, R. A.: Decision Analysis: Perspectives on Inference, Decision, and Experimentation, Proc. IEEE, 58, 5 (1970), pp. 632-643.

19) Messac, A.: Physical Programming-effective Optimization for Computational Design, AIAA J., 34 (1996), pp. 149-158.

20) Sanchis, J., Martínez, M. A., and Blasco, X.: Integrated Multiobjective Optimization and a Priori Preferences Using Genetic Algorithms, Information Sci., 178 (2008), pp. 931-951.

21) Planet Pulse | Flock 3p, Available from: https://www.planet.com/ pulse/tag/flock-3p/ (accessed April 17, 2017).

22) NASA - NSSDCA - Spacecraft - Details, https://nssdc.gsfc.nasa.gov/ nmc/spacecraftDisplay.do?id=2004-018A (accessed April 16, 2017).

S. Matunaga Associate Editor 